\title{
GROUP RINGS WHICH ARE AZUMAYA ALGEBRAS
}

\author{
BY
}

F. R. DEMEYER AND G. J. JA.NUSZ

\begin{abstract}
The group ring $R G$ of a group $G$ over a ring $R$ (with identity $l(R)$ ) is a separable algebra over its center if and only if the following conditions hold:

(a) $R$ is a separable algebra over its center:

(b) the center of $G$ has finite index in $G$ :

(c) the commutator subgroup $G^{\prime}$ of $G$ has finite order $m$ and $m l(R)$ is invertible in $R$
\end{abstract}

1. Introduction. If $R$ is a commutative ring and $G$ a finite group of order $n$, then the group ring $R G$ is a separable $R$-algebra if and only if $n 1(R)$ is invertible in $R$ $(1(R)$ is the identity of $R$ ). This is a well-known generalization of Maschke's theorem, a proof of which may be based on the ideas in [3, p. 41]. A ring is an Azumaya algebra if it is separable over its center. In particular, if $n 1(R)$ is invertible in $R$, then $R G$ is an Azumaya algebra. This condition is not necessary however. For example, if $G$ is any abelian group and $R$ any commutative ring then $R G$ is an Azumaya algebra. A less trivial example was provided by C. T. C. Wall in [7]. He showed that $R G$ is a direct sum of matrix rings over commutative rings when $R$ is a ring of $p$-adic integers and $G$ is a semidirect product of a finite cyclic group of order not divisible by $p$ and a finite, abelian $p$-group. In particular, $R G$ is an Azumaya algebra in this case.

The purpose of this paper is to prove the following theorem which determines which group rings $R G$ are Azumaya algebras.

THEOREM I. Let $R$ be a ring with identity $1(R)$ and let $G$ be a group. The group ring $R G$ is an Azumaya algebra if and only if the following conditions are satisfied:

(a) $R$ is an Azumaya algebra;

(b) the center of $G$ has finite index in $G$;

(c) the commutator subgroup $G^{\prime}$ of $G$ has finite order $m$ and $m 1(R)$ is invertible in $R$.

It seems worthwhile to explicitly state the theorem in the classical case for comparison with Maschke's theorem.

COROLlaRY. Let $F$ be a field and $G$ a finite group. Then the group algebra $F G$ is an Azumaya algebra if and only if the order of the commutator subgroup of $G$ is invertible in $F$.

Received by the editors November 8, 1982.

1980 Mathematics Subject Classification. Primary 16A16; Secondary 16A26, 16A27. 
For a prime $p$, the condition that $p$ does not divide the order of the commutator subgroup of the finite group $G$ is equivalent to the condition that $G$ is a finite group with an abelian $p$-Sylow subgroup and a normal $p$-complement. Our main result yields the following generalization of Wall's result mentioned above.

COROLlaRY. Let $R$ be a complete local ring with residue field of characteristic $p>0$, and let $G$ be a finite group. Then $R G$ is a direct sum of matrix rings over commutative rings if and only if $G$ has an abelian p-Sylow subgroup and a normal p-complement.

It should be remarked here that for a field $F$ of characteristic $p$ and a finite group $G$, the group algebra $F G$ is an Azumaya algebra if and only if it is isomorphic to a direct sum of matrix rings over commutative rings.

Our main reference for the theory of separable algebras is [3]. The reader may consult [1] for additional facts about separability.

2. Preliminary reductions. In this section we reduce the proof of Theorem I to the case of group algebras over fields.

Lemma 2.1. Let $A$ be a separable algebra over the commutative ring $R$ and let $\phi$ be $a$ ring epimorphism of $A$ onto a ring $B$. Then $\phi$ maps the center of $A$ onto the center of $B$; $B$ is a separable $\phi(R)$-algebra and an Azumaya algebra.

Proof. Since $\phi$ is an epimorphism, $\phi(R)$ is contained in the center of $B$. Thus $B$ is an $R$-algebra by way of $\phi$. The lemma follows from Propositions II.1.11 and II.1.12 of [3].

Lemma 2.2. Let $R$ be a ring with center $C$ and $G$ any group. Then $R G$ is an Azumaya algebra if and only if both $R$ and $C G$ are Azumaya algebras.

Proof. Suppose $R G$ is an Azumaya algebra. The homomorphism mapping the group $G$ onto the group of order one induces an epimorphism of $R G$ onto $R$. Hence $R$ is an Azumaya algebra.

Let $Z$ denote the center of $C G$. Then we have isomorphisms

$$
R G \cong R \otimes_{C} C G \cong\left(R \otimes_{C} Z\right) \otimes_{Z} C G .
$$

Proposition II.1.8 of [3] implies that $C G$ is an Azumaya algebra.

Conversely, suppose both $R$ and $C G$ are Azumaya algebra. Then the isomorphism $R G \cong R \otimes_{C} C G$ implies $R G$ is an Azumaya algebra.

This first reduction allows us to restrict our attention to the case of commutative coefficient rings.

For a ring $S$, let $z(S)$ denote the center of $S$.

LEMMA 2.3. Let $A$ be a commutative ring and $B$ a subring. For any group $G$ there is an isomorphism $A G \cong z(A G) \otimes_{z(B G)} B G$.

Proof. The center of $A G$ is the free $A$-module generated by the class sums $c(x)=\Sigma g x g^{-1}$, where $x$ is an element of $G$ with only a finite number of $G$ conjugates and the sum is over the distinct conjugates. Clearly these sums also freely generate the center $z(B G)$ as a $B$-module. Thus $z(A G)$ can be identified with $A \otimes_{B} z(B G)$. 
The lemma is a consequence of the following isomorphisms:

$$
A G \cong A \otimes_{B} B G \cong\left(A \otimes_{B} z(B G)\right) \otimes_{z(B G)} B G \cong z(A G) \otimes_{z(B G)} B G .
$$

Lemma 2.4. Let $F$ and $L$ be fields having the same characteristic and let $G$ be any group. Then FG is an Azumaya algebra if and only if $L G$ is an Azumaya algebra.

Proof. Assume $F G$ is an Azumaya algebra and let $K$ be the prime subfield of $F$. Then $z(K G)$ is a direct summand of $z(F G)$ as $z(K G)$-modules, so it follows from Lemma 2.3 and Proposition II.1.8 of [3] that $K G$ is an Azumaya algebra. Since $F$ and $L$ have the same characteristic, $K$ may be identified with a subfield of $L$. Then by applying Lemma 2.3 to $K \subseteq L$ we obtain that $L G$ is an Azumaya algebra.

Definition. For a commutative ring $R$, the characteristic set $\mathrm{CH}(R)$ is the set of nonnegative integers $q$ such that there exists an ideal $M$ of $R$ with $R / M$ an integral domain of characteristic $q$.

Proposition 2.5. Let $R$ be a commutative ring and $G$ any group. The group ring $R G$ is an Azumaya algebra if and only if for each integer $p$ in $\mathrm{CH}(R)$ there is some field $F$ of characteristic $p$ for which $F G$ is an Azumaya algebra.

Proof. Suppose $R G$ is an Azumaya algebra. Let $D=R / M$ be a domain of characteristic $p$, and let $F$ be its quotient field. Then $D G$ is an Azumaya algebra because it is a homomorphic image of $R G$. After applying Lemma 2.3 to $F \supseteq D$, we obtain $F G$ is also an Azumaya algebra. Thus the required fields exist.

Now suppose the required fields exist. In order to show $R G$ is an Azumaya algebra, it is sufficient to show $R G / M R G$ is separable over $z(R G) / M$ for every maximal ideal $M$ of $z(R G)$ [3, Theorem II.7.1]. So assume $M$ is such an ideal and let $L$ denote the field $z(R G) / M$. The inclusion of $R$ into $z(R G)$ implies that $R$ maps into $L$, so the characteristic of $L$ is an element of $\mathrm{CH}(R)$. By assumption there is some field $F$ having the same characteristic as $L$ such that $F G$ is an Azumaya algebra. By Lemma $2.4 L G$ is an Azumaya algebra.

Now let $\phi$ denote the composite of the following ring epimorphisms:

$$
L G \rightarrow L \otimes_{R} R G \rightarrow L \otimes_{z(R G)} R G \rightarrow R G / M R G .
$$

The last map in this sequence carries $a \otimes b$ to $a(b+M R G)$. For any $x$ in $G$ having finitely many $G$-conjugates, the class sum $c(x)$ is mapped by $\phi$ to $c(x)+M R G$. It follows then that $\phi(z(L G))=(z(R G)+M R G) / M R G$, which in turn is the image of $z(R G) / M$. Since $L G$ is separable over $z(L G), R G / M R G$ is separable over $z(R G) / M$ by Lemma 2.1 . This completes the proof.

This reduces the proof of the main theorem to the case in which the coefficient ring is a field. Notice that the statement "the order of $G^{\prime}$ is invertible in $R$ " is equivalent to the statement "no prime divisor of the order of $G^{\prime}$ is in $\mathrm{CH}(R)$ ".

3. The finite case. Throughout this section we consider the group ring $F G$ of a finite group $G$ over a field $F$.

If $F$ has characteristic zero then $F G$ is separable over $F$ and so $F G$ is an Azumaya algebra. So we restrict our attention to the case in which $F$ has characteristic $p>0$. In view of the interpretation of the condition that $p$ does not divide the order of $G^{\prime}$, the result to be proved is the following. 
THEOREM 3.1. Let $F$ be a field of characteristic $p>0$ and $G$ a finite group. Then $F G$ is an Azumaya algebra if and only if $G$ has an abelian $p$-Sylow subgroup and a normal $p^{\prime}$-complement.

Proof. Suppose $F G$ is an Azumaya algebra. In view of Lemma 2.4, we may assume $F$ is a finite splitting field for all subgroups of $G$. Since $z(F G)$ is a commutative, finite-dimensional $F$-algebra, it decomposes as $z(F G)=S_{1} \oplus \cdots \oplus S_{n}$ with each summand a finite local $F$-algebra. An Azumaya algebra over a finite ring is Morita equivalent to its center because the Brauer group of a finite ring is trivial [1]. Morita equivalence respects direct sums, thus each $p$-block $F G S$ of $F G$ is Morita equivalent to its center $S$. An Artinian local $F$-algebra has only one simple module and any Morita equivalent ring must have only one simple module also. Thus each $p$-block of $F G$ has only one simple module. By Corollary 65.3 of [4], it follows that $G$ has a normal $p$-complement $N$. Thus $G=N P$ with $P$ a $p$-Sylow subgroup of $G$. It is necessary to show that $P$ is abelian.

Since $P$ is a homomorphic image of $G$, and $F G$ is an Azumaya algebra, it follows that $F P$ is an Azumaya algebra. The $\operatorname{radical}, \operatorname{rad}(F G)$, of $F G$ is the ideal having the elements $1-x$ as basis for $x$ in $P$. By [3, Corollary II.3.7] there is an ideal $I$ of $z(F P)$ such that $\operatorname{rad}(F P)=I F P$. We now have the vector space sums

$$
F P=F+\operatorname{rad}(F P)=z(F P)+\operatorname{rad}(F P)=z(F P)+I F P .
$$

Since $I$ is a nilpotent ideal, Nakayama's lemma implies $F P=z(F P)$ and so $P$ is abelian.

The converse is well known and, as pointed out by the referee, is probably due to Morita [5]. A slightly more precise version appears in [6, p. 232]. It is shown that if $G=N P$, with $N$ a normal $p^{\prime}$-subgroup and $P$ an abelian $p$-group, and if $F$ is a perfect splitting field of characteristic $p$, then $F G$ is isomorphic to a direct sum of matrix rings over commutative rings of the form $F Q$ for $Q$ a subgroup of $P$.

4. The infinite case. In this section we allow the group $G$ to be infinite. The results to this point show that Theorem I will follow as a consequence of the next result.

THEOREM 4.1. Let $F$ be either a finite field or the field of rational numbers and let $G$ be a group. Then FG is an Azumaya algebra if and only if:

(i) the center of $G$ has finite index in $G$; and

(ii) the commutator subgroup $G^{\prime}$ has finite order $m$, and $m$ is invertible in $F$.

The proof is given in several steps. Denote the center of $G$ by $Z(G)$.

Lemma 4.2. Let $G=Z(G) H$ for some subgroup $H$ of $G$ and let $R$ be any commutative ring. Then $R G$ is an Azumaya algebra if and only if $R H$ is an Azumaya algebra.

Proof. Let $T$ be a set of elements in $Z(G)$ which represent the cosets of $H$ in $G$. Any element $g$ in $G$ has a unique representation $g=h t$ with $t$ in $T$ and $h$ in $H$. Moreover $g$ and $h$ have the same number of conjugates. If this number is finite the class sums satisfy $c(g)=c(h) t$. Since these finite class sums give an $R$-basis for the center, we obtain $z(R G)=\sum z(R H) t$, the sum taken over $T$. The coset decomposition implies $R G=\Sigma R H t$. From these equations we conclude $R G=z(R G) \otimes R H$, 
with the tensor product over $z(R H)$. Since $z(R H)$ is a direct summand of $z(R G)$, the equivalence stated in the lemma follows from Corollaries II.1.7 and II.1.10 of [3].

Lemma 4.3. Let $G$ be a group whose center has finite index. Then there is a finitely generated subgroup $H$ of $G$ with $G=Z(G) H$. Any such subgroup $H$ satisfies $H^{\prime}=G^{\prime}$.

Proof. As a generating set for $H$, take one element from each coset of $Z(G)$ in $G$. If $z$ and $w$ are central and if $x$ and $y$ are arbitrary elements of $G$, then the commutator of $z x$ and wy equals the commutator of $x$ and $y$.

Now we begin the proof that (i) and (ii) in Theorem 4.1 imply $F G$ is an Azumaya algebra. The proofs are different in the characteristic zero case and the characteristic nonzero case.

Proposition 4.4. If $F$ is a field of characteristic zero and if $G / Z(G)$ is finite, then $F G$ is an Azumaya algebra.

Proof. Separability over $z(F G)$ is equivalent to the existence of a separability idempotent $e$ in $F G \otimes_{z(F G)} F G^{0}$ [3, Proposition II.1.1]. We construct the element. Let $T$ be a set of representatives of the cosets of $Z(G)$ in $G$. Let

$$
e=[G: Z(G)]^{-1} \sum t \otimes t^{-1} .
$$

Then $e$ maps to 1 under the multiplication map. It is only necessary to show $(a \otimes 1) e=(1 \otimes a) e$ for each $a$ in $F G$. It is sufficient to show this for each $a$ in $G$. For any $t$ in $T$ there is some $c$ in $Z(G)$ and $s$ in $T$ with $a t=s c$. Thus

$$
(a \otimes 1)\left(t \otimes t^{-1}\right)=s c \otimes t^{-1}=s \otimes c t^{-1}=s \otimes s^{-1} a=(1 \otimes a)\left(s \otimes s^{-1}\right) .
$$

As $t$ runs through the elements of $T$, so does $s$. It follows that $e$ has the required properties and $F G$ is an Azumaya algebra.

We may now restrict our attention to the case where $F$ is the field of $p$ elements (or any finite field of characteristic $p$ ). We assume that (i) and (ii) of Theorem 4.1 hold. In view of Lemmas 4.2 and 4.3 we may also assume that $G$ is finitely generated.

Let $M$ be a maximal ideal of $z(F G)$. We intend to show $F G / M F G$ is separable over $z(F G) / M$. This is sufficient to prove $F G$ is an Azumaya algebra. The first step is to locate a subgroup of $Z(G)$ having some special properties.

LEMma 4.5. In the context just described, there is a subgroup $U$ of $Z(G)$ with the following properties:

1. $G / U$ is a finite group with commutator subgroup isomorphic to $G^{\prime}$.

2. The group ring $F(G / U)$ has $F G / M F G$ as an epimorphic image by a map which carries $z(F(G / U))$ onto $(z(F G)+M F G) / M F G$.

Proof. Since $G$ is finitely generated and $Z(G)$ has finite index, $Z(G)$ is a finitely generated abelian group [6, p. 177]. The structure theory for such group yields a decomposition

$$
Z(G)=B \times E=B \times\left\langle x_{1}\right\rangle \times \cdots \times\left\langle x_{n}\right\rangle
$$

with $B$ a finite group and $E$ a free abelian group. Let $T$ be a set of representatives for the cosets of $E$ in $G$. Then $T$ is a finite set. Let $c(t)$ denote the sum of the 
$G$-conjugates of $t$. Note that all elements of $G$ have only a finite number of conjugates because $Z(G)$ has finite index in $G$. Now we have a decomposition $z(F G)=\sum F E c(t)$.

The group ring $F E$ is the $F$-algebra generated by the $2 n$ elements $x_{i}, x_{i}^{-1}$, for $1 \leqslant i \leqslant n$. It follows that $z(F G)$ is a homomorphic image of a polynomial ring over $F$ in some finite number of variables; that is, $z(F G)$ is a finitely generated $F$-algebra. Now let $L$ denote the field $z(F G) / M ; L$ is a finitely generated algebra over $F$. This can happen only when $L$ is algebraic over $F[8$, p. 165]. Since $F$ is a finite field, $L$ is also finite. The inclusion of $E$ into $z(F G)$ induces a homomorphism of $E$ into the multiplicative group of $L$. Let $U$ denote the kernel of this homomorphism on $E$. Then $U$ has finite index in $E$ and so $G / U$ is finite. The group $U$ is torsion free since it is a subgroup of the torsion free group $E$. This implies $U \cap G^{\prime}=1$ because $G^{\prime}$ is finite. This implies $(G / U)^{\prime}=U G^{\prime} / U=G^{\prime}$, and this proves statement 1 .

The natural projection $\phi^{\prime}$ of $F G$ onto $F G / M F G$ has $u-1$ in its kernel for each $u$ in $U$. Hence $\phi^{\prime}$ factors through $F(G / U)$ and induces an epimorphism $\phi$ from $F(G / U)$ onto $F G / M F G$. In order to verify the assertion about the centers, we begin with the following observation:

If $x$ and $y$ are distinct conjugates in $G$ then $x U$ and $y U$ are distinct conjugates in $G / U$.

This is an immediate consequence of the equation $U \cap G^{\prime}=1$. This implies that a class sum $c(x)$ in $F G$ is mapped onto a class sum $c(x U)$ in $F(G / U)$ by the natural projection. It follows that $\phi(z(F(G / U)))=\phi^{\prime}(z(F G))$, and statement 2 is proved.

Now the pieces may be assembled. The order of $(G / U)^{\prime}$ is invertible in $F$ by Lemma 4.5 and so $F(G / U)$ is Azumaya algebra by Theorem 3.1. Thus its epimorphic image $F G / M F G$ is separable over the image of the center of $F(G / U)$. This image is $(z(F G)+M F G) / M F G$, which is also the image of $z(F G) / M$. Hence $F G / M F G$ is separable over $z(F G) / M$. This completes the proof that (i) and (ii) of Theorem 4.1 imply that $F G$ is an Azumaya algebra.

Now we begin the proof of the converse. It is assumed that $F$ is a field and that $F G$ is an Azumaya algebra. The proof that (i) and (ii) hold relies upon the results and ideas of Passman [6]. The first important concept is that of the finite conjugacy subgroup of $G$, which is denoted by $\Delta(G)$, or just $\Delta$ if there is no danger of confusion. This is the set of all elements of $G$ which have only a finite number of conjugates. One may verify that $\Delta(G)$ is a normal subgroup of $G$ [6, Chapter 4].

LEMMA 4.6. If $F$ is a field and $G$ a group such that $F G$ is an Azumaya algebra, then $G=\Delta(G), G^{\prime}$ is finite, and the center $Z(G)$ has finite index in $G$.

Proof. Let $Z$ denote $z(F G)$. Then $F G$ is a finitely generated projective $Z$-module [3, Theorem II.3.4]. Also $Z$ is a subring of $F \Delta$ since the class sums $c(g)$ span $Z$ as $g$ runs through $\Delta$. Let $T$ be a set of coset representations of $\Delta$ in $G$. Then $F G=\Sigma F \Delta t$ is a direct decomposition. Since $F G$ is finitely generated over a subring of $F \Delta$, it follows that the group $D=G / \Delta$ is finite. Now there is a canonical map of $F G$ onto $F D$ which maps the center $Z$ onto the center of $F D$ by Lemma 2.1. However this map carries $\Delta$ onto 1 and thus carries $Z$ onto $F$. The center of the group algebra of a finite group $D$ has dimension one only when $D=1$. Thus $G=\Delta$. 
Now let $S$ be a finite set of elements in $G$ for which $F G=\Sigma Z s, s$ in $S$. Then $C_{G}(s)$ has finite index in $G$ and, thus, $H=\cap C_{G}(s)$ also has finite index in $G$. It is clear that any element of $H$ centralizes all of $F G$, and so $H=Z(G)$, and $Z(G)$ has finite index. Finally a lemma of Schur [6, p. 155] implies $G^{\prime}$ is finite.

All that remains to show is that the order of $G^{\prime}$ is invertible in $F$. There is a finitely generated subgroup $H$ with $G=Z(G) H, H^{\prime}=G^{\prime}$ (Lemma 4.3) and $F H$ an Azumaya algebra (Lemma 4.2). Moreover there is a torsion free subgroup $E$ of $Z(H)$ such that $H / E$ is finite with commutator subgroup isomorphic to $G^{\prime}$ (by the proof of Lemma 4.5). It follows that $F(H / E)$ is an Azumaya algebra and so, by Theorem 3.1, the order of $G^{\prime}$ is invertible in $F$.

This completes the proof of the main result.

\section{REFERENCES}

1. M. Auslander and O. Goldman. The Brauer group of a commutative ring. Trans. Amer. Math. Soc. 97 (1960), 367-4(09.

2. C. W. Curtis and I. Reiner, Representation theory of finite groups and associative algebras, Interscience, New York, 1962.

3. F. DeMeyer and E. Ingraham, Separable algehras over commutative rings, Lecture Notes in Math., vol. 181, Springer, Berlin and New York, 1971.

4. L. Dornhoff, Group representation theory, Part B, Dekker, New York, 1972.

5. K. Morita, On group rings over a modular field, Sci. Rep. Tokyo Bunrika Daigaku 4 (1951), 177- 194.

6. D. S. Passman, Algehraic structure of group rings, Wiley, New York, 1977.

7. C. T. C. Wall, Norms of units in group rings, Proc. London Math. Soc. (3) 29 (1974), 593-632.

8. O. Zariski and P. Samuel, Commutative algehra, Vol. II, Van Nostrand, Princeton, N. J., 1960.

Department of Mathematics, Colorado State University, Fort Collins, Colorado 80523

Department of Mathematics, University of Illinois, Urbana, Illinois 61801 